\title{
BMJ Open Physical activity trails in an urban setting and cardiovascular disease morbidity and mortality in Winnipeg, Manitoba, Canada: a study protocol for a natural experiment
}

Erin Hobin, ${ }^{1}$ Anders Swanson, ${ }^{2}$ Gillian Booth, ${ }^{3}$ Kelly Russell, ${ }^{4}$ Laura C Rosella (i) , ${ }^{1}$ Brendan T Smith, ${ }^{1}$ Ed Manley, ${ }^{5}$ Wanrudee Isaranuwatchai, ${ }^{6}$ Stephanie Whitehouse, ${ }^{7}$ Nicole Brunton, ${ }^{4}$ Jonathan McGavock (1) ${ }^{4}$

To cite: Hobin E, Swanson A, Booth G, et al. Physical activity trails in an urban setting and cardiovascular disease morbidity and mortality in Winnipeg, Manitoba, Canada: a study protocol for a natural experiment. BMJ Open 2020;10:e036602. doi:10.1136/ bmjopen-2019-036602

\section{- Prepublication history and} additional material for this paper are available online. To view these files, please visit the journal online (http://dx.doi. org/10.1136/bmjopen-2019036602).

Received 03 January 2020 Revised 23 January 2020 Accepted 03 February 2020
Check for updates

(C) Author(s) (or their employer(s)) 2020. Re-use permitted under CC BY-NC. No commercial re-use. See rights and permissions. Published by BMJ.

For numbered affiliations see end of article.

Correspondence to Dr Jonathan McGavock; jmcgavock@chrim.ca

\section{ABSTRACT}

Introduction Aspects of the built environment that support physical activity are associated with better population health outcomes. Few experimental data exist to support these observations. This protocol describes the study of the creation of urban trials on cardiovascular disease (CVD)-related morbidity and mortality in a large urban centre.

Methods and analysis Between 2008 and 2010, the city of Winnipeg, Canada, built four, paved, multiuse (eg, cycling, walking and running), two-lane trails that are $5-8 \mathrm{~km}$ long and span $\sim 60$ neighbourhoods. Linking a population-based health data with census and environmental data, we will perform an interrupted time series analysis to assess the impact of this natural experiment on CVD-related morbidity and mortality among individuals $30-65$ years of age residing within 400-1200 $\mathrm{m}$ of the trail. The primary outcome of interest is a composite measure of incident major adverse CVD events (ie, CVD-related mortality, ischaemic heart disease, stroke and congestive heart failure). The secondary outcome of interest is a composite measure of incident CVD-related risk factors (ie, diabetes, hypertension and dyslipidaemia). Outcomes will be assessed quarterly in the 10 years before the intervention and 5 years following the intervention, with a 4-year interruption. We will adjust analyses for differences in age, sex, ethnicity, immigration status, income, gentrification and other aspects of the built environment (ie, greenspace, fitness/recreation centres and walkability). We will also assess trail use and trail user profiles using field data collection methods.

Ethics and dissemination Ethical approvals for the study have been granted by the Health Research Ethics Board at the University of Manitoba and the Health Information Privacy Committee within the Winnipeg Regional Health Authority. We have adopted an integrated knowledge translation approach. Information will be disseminated with public and government partners.

Trial registration number NCT04057417.

\section{RATIONALE}

The built environment is at the interface of public and health policy. ${ }^{12}$ Social scientists
Strengths and limitations of this study

- We are relying on natural experiment consisting of the expansion of trails within an urban environment to test our research hypothesis.

- We have access to data for the entire urban population over a 20 -year time frame, capturing all healthrelated endpoints.i

- We are triangulating health outcome data with robust field data on trail use to describe the population impact of the trail expansion.

- We cannot determine individual-level physical activity levels in persons living within intervention neighbourhoods.

- We will not be able to capture individual-level trail use and therefore do no know what percentage of the population within each neighbourhood are using the trails.

have explored the association between the environment and health for decades, ${ }^{3}$ and a growing body of evidence show some aspects of the built environment are associated with cardiovascular disease $(\mathrm{CVD})^{3}$ and its preceding risk factors. ${ }^{4-8}$ The favourable associations between aspects of the built environment and CVD-related health have been observed primarily through the promotion of physical activity.

Various elements of the built environment are associated with increased physical activity, particularly access to walkable neighbourhoods and green space, and the proximity of recreation facilities. ${ }^{2} 4$ Importantly, individuals living in built environments that facilitate physical activity (eg, greater access to walkable neighbourhoods and green space) display more favourable cardiovascular health profiles. ${ }^{2910}$ For example, individuals 
are $10 \%-20 \%$ more likely to meet daily recommendations for physical activity and 5\% less likely to have obesity for every incremental increase in access to recreational facilities, green space and walkable neighbourhoods. ${ }^{211}$ Conversely, individuals living in environments with more fast food outlets and fewer healthy food options are more likely to eat unhealthy food and live with obesity or diabetes. ${ }^{12} 13$ Based on these associations, changes to the built environment are projected to be a cost-effective population health intervention to promote physical activity and prevent CVD. ${ }^{14}{ }^{15}$ Unfortunately, there are limited studies of the built environment and CVD-related morbidity and mortality. Additionally, current evidence in this field largely draws on underpowered cross-sectional studies. ${ }^{46}$ While associations between the built environment and health behaviours seem robust, it remains unclear if changes to the built environment are associated with improved health outcomes. Accordingly, experimental data are needed to determine if, and the extent to which, changes to the built environment are associated with reduced CVD-related morbidity and mortality. ${ }^{17-19}$

Elements of the built environment that support physical activity include neighbourhood walkability, access to greenspace and proximity to fitness/recreation centres. ${ }^{2}$ Urban multiuse trails are an additional, but poorly studied, aspect of the built environment that could support greater population-level daily physical activity. ${ }^{20}$ Urban trails are multiuse protected areas for cycling alone or a combination of cycling, walking and running. They can be used to facilitate active transportation or a combination of active transport and recreational physical activity. Despite widespread growth of trail networks in urban areas, they are not included in calculations of walkability within a neighbourhood, ${ }^{21}$ and little information exists for their association with health outcomes.

As randomised controlled trials are virtually impossible in this field, natural experiments provide the only feasible opportunity to generate experimental evidence to examine a potential causal link between the built environment and health outcomes. The most recent systematic review and meta-analysis investigating the influence of the built environment on health outcomes found no experimental studies, and none of the studies included disease-specific CVD or CVD-related outcome measures (ie, mortality, myocardial infarction and/or comorbid conditions)..$^{9}$ Consistent with the results of observational studies examining associations between the built environment and health, the majority of natural experiments revealed improvements in more proximal measures of CVD, for example, physical activity; however, there is no experimental evidence examining whether changes in the built environment lead to changes in CVD-related morbidity or mortality. ${ }^{9}$

The current study describes the methods and protocol for a natural experimental study evaluating the impact of a large expansion of an urban trail network on CVDrelated morbidity and mortality in a large urban centre in Canada.

\section{METHODS AND ANALYSIS}

\section{Study aims and hypotheses}

The primary objective of this natural experimental study is to capitalise on major changes to the built environment that occurred in Winnipeg, Canada, between 2010 and 2014 to determine if a significant expansion of an urban trail network is associated with reduced CVD-related morbidity and mortality. The secondary aim of the study is to determine the cost-benefit ratio of this intervention compared with forecasted costs associated without the expansion of the urban trail network. The last aim of the study is to describe patterns of trail use and trail users during the intervention time period. Our primary study hypothesis is that an expansion of an urban trail network will reduce population-level CVD-related mortality and morbidity in neighbourhoods within $400-1200 \mathrm{~m}$ of the new trail compared with neighbourhoods lying outside these boundaries.

\section{Study design}

The most robust approach to test this research hypothesis is a quasiexperimental interrupted time series analysis with a comparison condition. The proposed interrupted time series analysis is strengthened by the use of a social and health data repository that provides an opportunity to link census, social and health data for the entire population of the City of Winnipeg beginning in 1995 . $^{22-27}$ Interrupted time series designs are considered the most valid quasiexperimental designs when a randomised controlled trial is not possible. ${ }^{28} 29$ They are the preferred design for population health interventions or pragmatic experiments as they strengthen pre-post designs, particularly if an appropriate comparison condition is available. ${ }^{28-30}$ This approach is considered the gold standard for a natural experiment, as it captures real-world changes in population-level health outcomes following large policy or practice changes ${ }^{31-34}$ that can eventually inform changes in health systems.

Using population-level outcome data over a 20-year period (2000-2019), we will construct an interrupted time series with a comparison condition. We selected a 20 -year time frame as it will provide $\sim 60$ data points $(\sim 40$ before and $\sim 20$ after the intervention) to test for changes in the slopes of CVD-related morbidity and mortality between groups before and after the expansion of urban trails. This design will allow us to better control for biases that accompany non-randomised study design ${ }^{35-37}$ such as (1) secular trends, which in this case may be changes in CVD rates that could be interpreted as intervention effects if comparison neighbourhoods were not available; (2) seasonal effects, as rates of CVD tend to be higher during winter months ${ }^{38}$; (3) duration of the intervention where trails may only be used in the first few months or years following construction and data collected in the year or two following the expansion would not have identified this effect; (4) random short-term fluctuations in CVD rates that may occur that do not reflect overall trends and can lead to biased intervention effect estimates if only 
a short time window is studied; and (5) autocorrelation where rates of CVD are likely to be associated between time points and between neighbourhoods. In addition to employing an interrupted time series design to minimise biases, we will employ a series of complimentary statistical features that include segmented time series regression techniques ${ }^{28} 37$ and autoregressive integrated moving average models. ${ }^{30} 39$ To improve comparability of treatment and comparison neighbourhoods, we will create balanced treatment and comparison groups using propensity score matching. ${ }^{40}$

\section{Study population}

This study will be conducted within the metropolitan area of Winnipeg, Manitoba, Canada's seventh largest urban centre. It is considered a slow growth city, relative to other major urban centres in Canada. ${ }^{41}$ Winnipeg includes $\sim 700000$ residents, representing $>50 \%$ of the population of the province of Manitoba. Data from administrative healthcare databases within the Manitoba Centre for Health Policy (MCHP) will be used to derive populationlevel estimates of CVD and CVD-related comorbid conditions as previously done. ${ }^{42-44}$ Health information for local residents will be linked to dissemination-level data using individual postal codes. We will restrict the analyses to the adult population aged $30-65$ years as previously done, ${ }^{21}$ as CVD endpoints are $<1 / 10000$ among persons under 30 years in Manitoba. ${ }^{42}$

\section{Intervention group: Winnipeg urban trail network expansion}

In the Canadian context, an urban trail is a multiuse public path that creates an attractive transportation and leisure activity corridor through a built environment, used largely during the summer months. ${ }^{45}$ Urban trails are an ideal component of the built environment to study with a quasiexperimental design and aggregated data. In contrast to more commonly studied aspects of the built environment that promote physical activity (eg, neighbourhood walkability and green space), trails are substantially easier to manipulate, as they require minimal space within the urban landscape and can affect a much larger segment of the urban population as they cross multiple neighbourhoods.

Between 2008 and 2010, the City of Winnipeg and Province of Manitoba invested $\$ 25$ million to expand the infrastructure for leisure and transportation-based physical activity. Several mixed socioeconomic neighbourhoods within Winnipeg were exposed to one of four new urban trails between 2008 and 2010, affecting roughly 250000 residents within $\sim 350$ dissemination areas. Details of the greenways are provided in table 1 , and their location within the city are provided in figure 1 . The four multiuse urban trails are over $5 \mathrm{~km}$ in length, paved, twolane paths that are cleared and maintained by the City of Winnipeg Department of Transportation 12 months of the year. They provide efficient and desirable trails for both active transportation and leisure physical activity. The majority of the population of Winnipeg do not live in dissemination areas that are within a reasonable distance to access the urban trails and therefore will be treated as the control condition (figure 1 - map).

\section{Outcome measures}

The primary outcome measure will be a composite endpoint of incident CVD-related events including new hospital admissions for cardiac-related events (CVDmortality, ischaemic heart disease and stroke) and CVDrelated comorbidities (table 2). ICD-9-CM codes will be used up to 1 April 2004 and ICD-10-CM codes afterwards

Table 1 Details of the intervention: new urban trail greenways

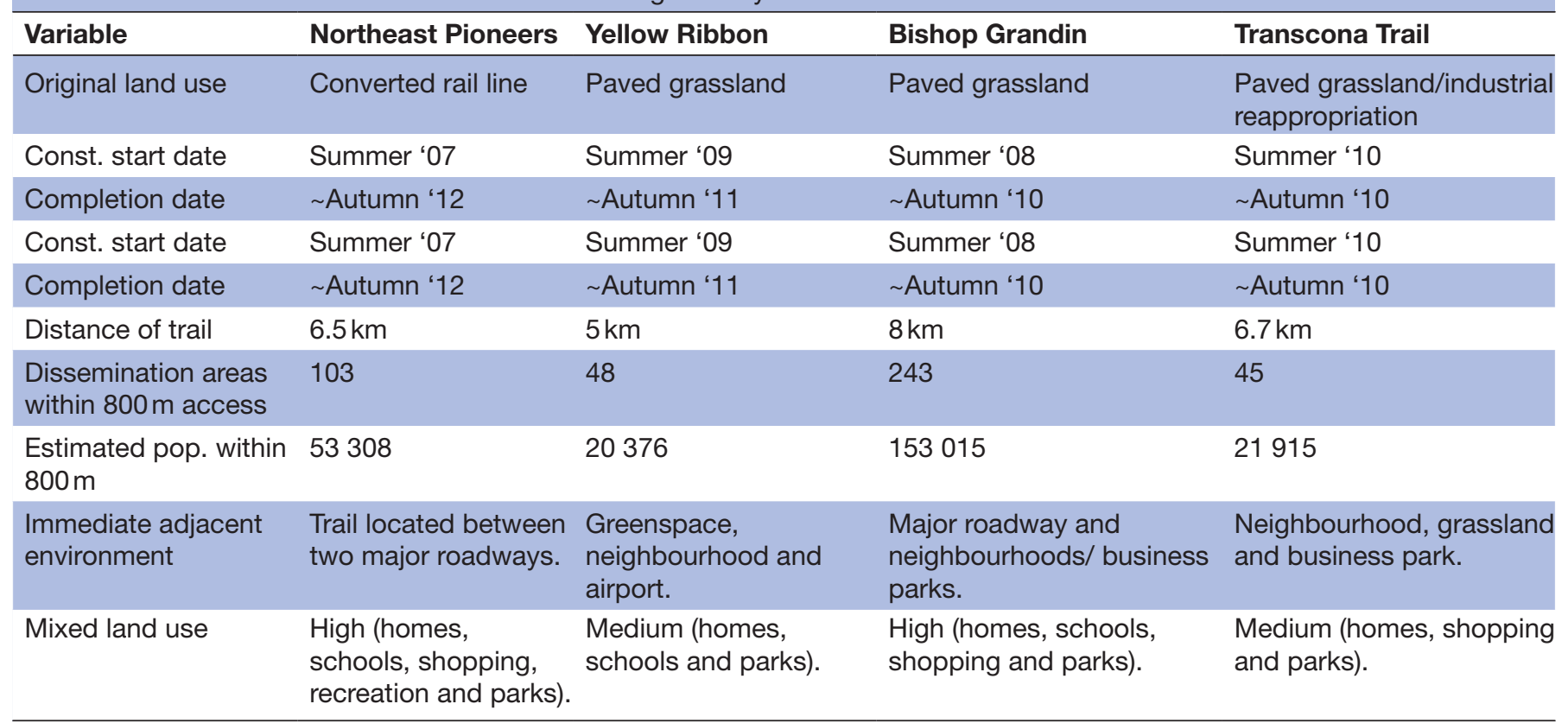




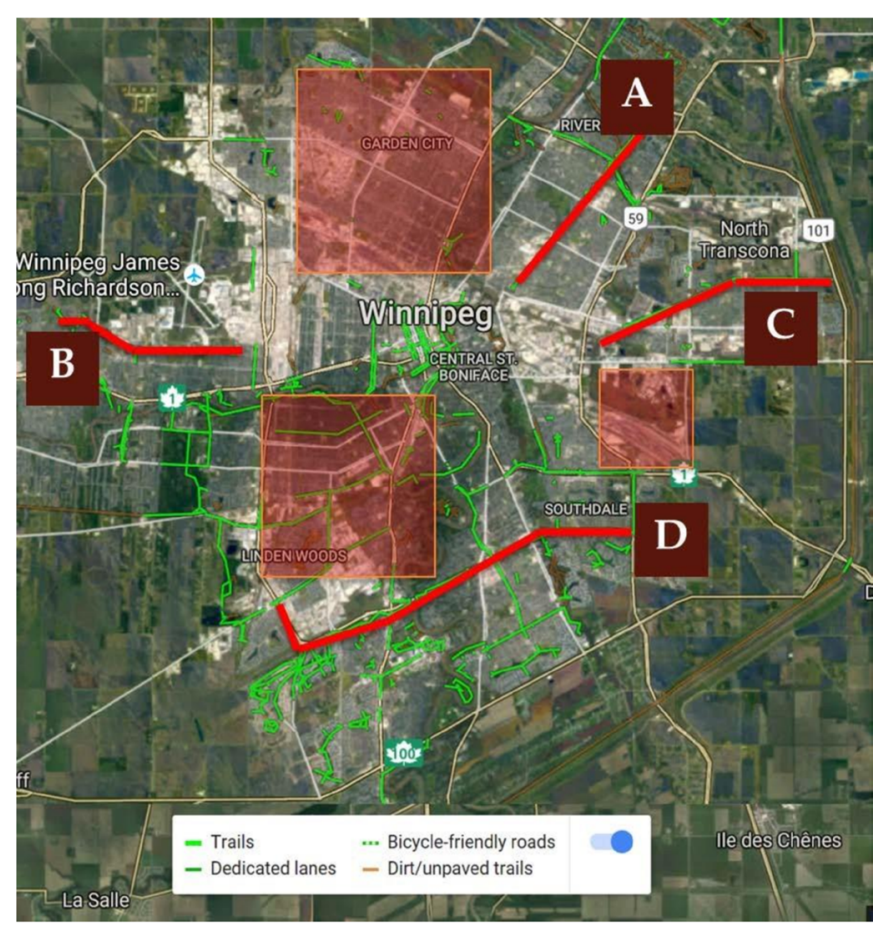

Complete trail map includes old (green lines) and new trails/greenways (red lines), comprising $400 \mathrm{~km}$ of total trails. Large red trails are "high dose" multi-use trails that extend across multiple neighbourhoods and accessible 12 months of the year $(A=$ Northeast Pioneers Greenway; $B=$ Yellow Ribbon Greenway; $C=$ Transcona Trail \& D= Bishop Grandin Greenway). Other green lines represent medium or low impact bike trails or gravel trails not used in winter or only for active commuting. Note large red areas that have not received trails.

Figure 1 Map of trail locations within Winnipeg, Manitoba, Canada, as of 2016.

for disease diagnosis (online appendix table 1). These endpoints were selected as they represent the largest burden of CVD-related morbidity in Canada, are the most relevant to policy makers and most likely to be modified by increased physical activity levels. These outcomes are derived from validated algorithms, providing an extremely accurate and sensitive capture of endpoints. ${ }^{43}$ Heart failure and peripheral artery disease will not be included in the composite outcome as algorithms have yet to be validated in the repository. The secondary outcome measure will be CVD-related comorbidities, including hypertension, diabetes and dyslipidaemia, as they are more proximal outcomes associated with changes in physical activity levels ${ }^{1221}$ and therefore more likely to change within neighbourhoods during the relatively short time frame of this intervention (table 2).

\section{EXPLORATORY AND CONFOUNDING VARIABLES \\ Trail usage}

Eco-Counter Dual Inductive Loop Zelts are automated cyclist counters that will be used to measure trail usage as an exploratory variable, between 2014 and 2019. ${ }^{47}$ Zelts have been discussed as an acceptable and reliable research tool to capture trail use. ${ }^{48}$ Zelts are comprised of a main unit and sensory wiring. The main unit includes a battery, Global System for Mobile transmission device and counting unit buried in manholes on the outer edge of a trail. Zelt counters will be placed at 10 locations across the five trail developments and will collect cyclist data for 24 hours/day, 7 days per week. Data collected from Zelts are time stamped and automatically uploaded to EcoVisio online software via cellular networks at 03:00 every day.

\section{Field data collection to survey trail users}

To determine trail user demographics, we will conduct two waves of intercept surveys among a convenience sample of 1000 trail users-one wave in 2018 and one in 2019. Users will be asked to complete a brief survey to provide demographic information, self-reported trail usage and the perceived impact of usage on both their physical and mental health. These surveys will be designed to be completed in 3-5 min, to minimise participant burden and facilitate the collection of responses from the highest possible number of users. Demographic data will include self-identified gender and ethnicity, age group, residential postal code, newcomer status and annual household income. Analyses of these data will determine which, if any, specific groups experience disproportionate access to the trails. Usage data will outline typical weekly trail use (in terms of frequency, duration and types of activities), commute time to reach trail paths and reasons for using the trail system. Survey collection will occur year-round when weather permits as either paper or technological survey instruments may not perform optimally in extreme winter conditions.

Survey participants will be asked to provide the first three digits of their postal code. This data will be used to geo-map the neighbourhoods of visiting users for each trail through dot density mapping techniques. Geo-maps will reflect the population distribution and demographic variation represented at each trail. Postal code data and produced geo-maps will also be combined with City of Winnipeg Census data (2016) to provide insight into represented residential neighbourhood distributions for ethnicity and household income.

\section{CONFOUNDING VARIABLES}

A detailed list of confounding variables and their source are provided in table 3 .

\section{Socioeconomic status (SES)}

Household income will be determined by census data, which are publicly available every 5 years. We will rely on data from the 2006 and 2016 surveys to assess household income. We will also rely on a validated continuous index of material and social deprivation (Socioeconomic Factor Index), which is calculated for each dissemination area in the province of Manitoba and reflects neighbourhoodlevel socioeconomic well-being. ${ }^{49}$

\section{Gentrification}

Gentrification describes demographic changes that may occur in a community over time as individuals with 
Table 2 Outcome measures in the natural experiment of urban trail expansion

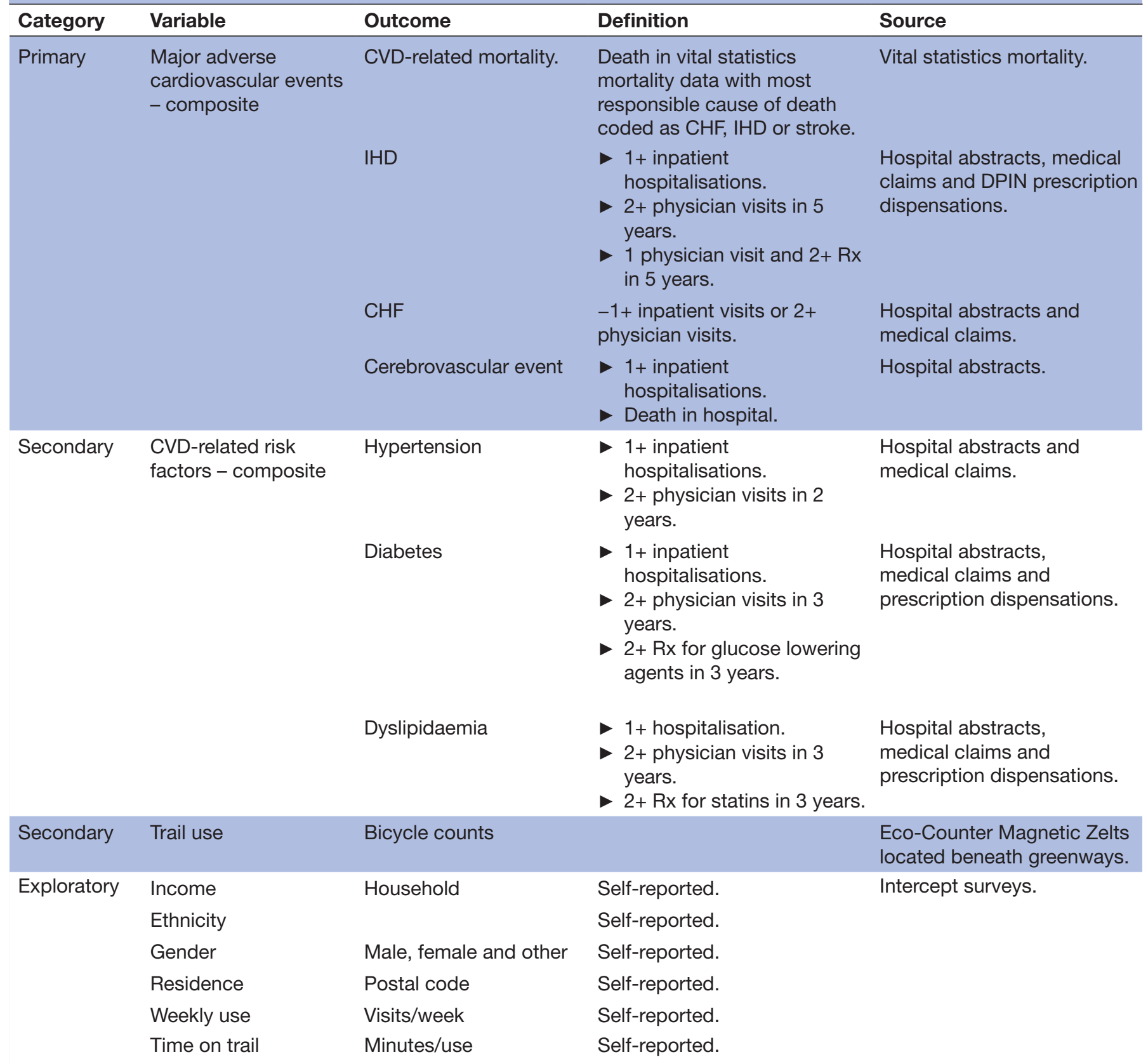

CHF, congestive heart failure; CVD, cardiovascular disease; DPIN, Drug Program Information Network; ICD, international classification code; $\mathrm{IHD}$, ischaemic heart disease; Rx, prescription.

a higher SES move into lower socioeconomic areas. ${ }^{50}$ Since SES can have large implications for outcomes related to $\mathrm{CVD},{ }^{51-53}$ diabetes $^{5154}$ and mortality, ${ }^{52}$ it will be important to account for gentrification within neighbourhoods. First, changes in property taxation between 2008 and 2018 assessed by the City of Winnipeg will be examined for each included neighbourhood to approximate increases or decreases in household value. Additionally, 2001 Canadian Census data and the 2016 National Household Survey data will be used to determine shifts in relevant indicators including poverty, education, unemployment rates, language barriers, average household income and ethnicity. Finally, data characterising retail indicators will be obtained from DMTI Spatial Inc (20062016) to compare the number and ratio of specialty coffee houses versus coffee and doughnut shops per capita within neighbourhoods. ${ }^{21}$ This will act as a surrogate variable representing demographic shifts in retail demands.

\section{Built environment determinants of physical activity}

The Canadian Urban Environmental Health Research Consortium (CANUE) is a multidisciplinary collaboration of specialists focused on environmental exposures and population health. ${ }^{56}$ CANUE integrates several 
Table 3 Potential sources of confounding between intervention and control groups that are available within linked databases

\begin{tabular}{|c|c|c|c|c|}
\hline Category & Variable & Definition & Source & Years available \\
\hline \multirow[t]{2}{*}{ Socioeconomic status } & Income & Household income & Census & 2006 and 2016 \\
\hline & SEFI & $\begin{array}{l}\text { Material and social } \\
\text { deprivation }\end{array}$ & MCHP repository & Annual \\
\hline \multirow[t]{2}{*}{ Demographics } & Age & & Census & 2006 and 2016 \\
\hline & Sex & & Census & 2006 and 2016 \\
\hline \multirow[t]{2}{*}{ Gentrification } & $\begin{array}{l}\text { Change in mean age } \\
\text { neighbourhood }\end{array}$ & $\begin{array}{l}\text { Taxation valuation of value } \\
\text { of property }\end{array}$ & City of Winnipeg & 2008 to 2018 \\
\hline & Property value & & & \\
\hline \multirow{2}{*}{$\begin{array}{l}\text { Built environmental } \\
\text { factors that support } \\
\text { physical activity }\end{array}$} & Greenspace & Distance to park (km) & DMTI/CANUE & 2006 and 2016 \\
\hline & Fitness/recreation centres & Distance to centre & CANUE & 2006 and 2016 \\
\hline
\end{tabular}

CANUE, Canadian Urban Environmental Health Research Consortium; DMTI, Digital Mapping company name; MCHP, Manitoba Centre for Health Policy; SEFI, Socioeconomic Factor Index.

population health databases and environmental exposure datasets into an openly available resource. From the CANUE dataset, we will integrate examine several estimates of greenspace, including accessibility of green space, type of vegetation, tree canopy cover in control for access to greenspaces in the treatment and comparison and intervention neighbourhoods across Winnipeg via the 2016 ArcGIS survey. All these data will be treated as confounding and adjusted for in the final analyses. We will also assess neighbourhood walkability using Canadian Active Living Environments (CAN-ALE) scores calculated from a GIS-based composite of population density, mixed land use, access to public transportation and street connectivity. Finally, we will estimate the number of fitness and recreation centres within $3 \mathrm{~km}$ of each household.

\section{Patient and public involvement}

Members of the public will be involved at several stages of the study. Members of the public were involved in developing the study design, in response to priorities established by a local organisation (Winnipeg Trails Association) dedicated to supporting urban trails for physical activity. Members of the public will help with recruitment of trail users during field data collection and collect survey responses. This organisation will also develop public and policy maker friendly tools to disseminate results of the study via their social media platforms and at local meetings. The organisation, represented by its executive director (AS), acted as a coapplicant on the grant and a collaborator throughout the project.

\section{POWER CALCULATION AND SAMPLE SIZE}

We have the advantage of leveraging a health data repository that includes the majority of residents in Winnipeg and captures all endpoints that occurred within each time point for residents in that neighbourhood. During the preintervention period (2006/2007 and 2011/2012), overall mortality, annual incident rates of ischaemic heart, hypertension and diabetes were nearly identical between treatment and comparison neighbourhoods. ${ }^{42}$ The stability of outcome measures will facilitate the detection of small, but meaningful, changes in trends or absolute events within treatment neighbourhoods. Segmented regression with propensity score matching will be used to compare changes in event rates between treatment and comparison neighbourhoods. The goal of the propensity score matching will be to pair the approximately 60 treated units (ie, neighbourhoods) with approximately 60 randomly selected comparison units balanced on key covariates known to influence physical activity levels and CVD-related mortality and morbidity (SES, age, ethnicity and walkability) permitting analysis of data as if it arose from a randomised design. ${ }^{57}$ Power analysis is based on a comparison of incidence rates in matched treated and comparison units.

Sample size calculations for clustered count data, where treatment assignment is at the neighbourhood level and the outcome is an incidence rate (eg, Poisson count), were used to estimate the minimal detectable effect size. ${ }^{18}$ We applied the Hayes-Donner method to estimate the number of neighbourhoods required to discern a given effect size, expressed as the difference between group-specific incidence rates under the assumption of 
a shared coefficient of variation across groups. These are conservative estimates, since they do not account for the paired nature of the data. ${ }^{58}$ Using preintervention neighbourhood incidence rates, ${ }^{42}$ we created a power plot of the minimal detectable effect based on number of neighbourhoods affected by trail expansion. Outcome-specific incidence rates were calculated from MCHP-led Health Atlases ${ }^{42} 59$ for 26 well-defined Winnipeg neighbourhoods between 2002 and 2007 (preintervention, crude rates per 100 person-years). Assuming an alpha $=0.05$, beta $=0.2$ and an average neighbourhood incidence rate for the composite outcome $\approx 4.95 \pm 0.45$ per 100 person years in treatment and comparison tracts, ${ }^{42}$ we are powered to detect a $10 \%$ difference in the primary outcome with 50 treatment neighbourhoods and $9 \%$ with 60 neighbourhoods. ${ }^{58}$ This effect size is relevant to stakeholders as it translates into 350 fewer CVD-related events annually within the city. The effect is conservative, as previous population interventions like smoking bans (17\% reduction in CVD events) ${ }^{60}$ new public transportation $(80 \% \text { difference in odds of obesity })^{61}$ or more walkable neighbourhoods (19\% reduced incidence of type two diabetes ${ }^{21}$ have yielded larger effects.

\section{PROPOSED ANALYSES}

To test the primary study hypothesis, that an expansion of an urban trail network will reduce population-level CVD-related mortality and morbidity in neighbourhoods within $400-1200 \mathrm{~m}$ of a new trail (intervention group), to a greater extent than neighbourhoods outside those boundaries, outcome data will initially be aggregated by neighbourhood and trends analysed for the entire study period. The two primary outcome measures, composite endpoints of incident major adverse CVD-related events and CVD-related risk factors will be treated as binary outcomes collected $\sim 36$ during the preintervention period and at 36 time points after the intervention (ie, trail expansion), following Cochrane recommendations. ${ }^{35}$ There will be a 2 -year ${ }^{35}$ lag incorporated into the time series reflecting the time during which the trails were being constructed. These time points reflect extensive literature showing that CVD-related behaviours ${ }^{62} 63$ and endpoints vary seasonally ${ }^{64}{ }^{65}$ and that the impact of natural experiments requires several years to be detected at the population level..$^{28} 3537$

\section{Time series analyses}

We will use two different time series methods to estimate the effect of the intervention on CVD-related endpoint incident rates in treatment neighbourhoods relative to trends among the comparison neighbourhoods. First, a multigroup segmented regression of interrupted time series data will be used to assess the effect of the intervention on CVD-related incidence, both immediately (change in level) and over time (change in trend) by creating indicator variables as described elsewhere. ${ }^{37} 40$ The level will be the base rate of CVD-related endpoints at the beginning of the preintervention period (2000) and the value immediately following each change point at which successive segments join until 2010. The trend is the rate of change of CVD-related endpoints (in other words, the slope) during a segment. Autoregressive errors will be modelled to account for correlated outcomes.

\section{Autoregressive integrated moving average (ARIMA)}

An ARIMA model will be fitted for the CVD incidence time series by using the standard approach for identification, estimation and checking. ${ }^{66}$ Trend and periodic seasonal terms will be applied to the entire study period (January 2000-July 2019). A separate ARIMA model will also be built for the preintervention period to forecast CVD evolution of the treated neighbourhoods. The number of CVD endpoints prevented by the intervention will be estimated by calculating the difference between the predicted number and the observed number of cases. Should we encounter difficulty fitting an ARIMA model to a relatively small dataset, we will rely on exponential smoothing models or the Holt Winters Algorithm. ${ }^{66}$ Although they require larger sample sizes, these methods are ideal for this project as (1) they permit a variety of different types of intervention effects to be modelled explicitly and (2) they are well suited to forecasting future trends.

\section{Cost-benefit analysis}

The cost-benefit analysis will focus on describing the 'value for money' of the intervention. The objective of the economic analysis is to compare the cost and effect of the treatment group relative to the comparison group data collected in the study. ${ }^{67}$ To align with the effectiveness analysis, the primary outcome variable in the economic analysis will be a composite endpoint of incident composite CVD-related endpoints, similar to previous studies by one of the team members. ${ }^{67-69}$ Costs for each participant will include those associated with the intervention (eg, trail construction and maintenance costs) and health service utilisation obtained from administrative data. From the perspective of the public payers (provincial and federal governments), we will conduct the economic analysis using the net benefit regression framework, ${ }^{70} 71$ which enables an adjustment for potential confounders. The main output of the economic analysis is the incremental net benefit of the treatment relative to the comparison group. In addition, we will estimate incremental costeffectiveness ratios that will represent an incremental cost per one incident CVD endpoint prevented or one CVDrelated comorbidity prevented. Furthermore, as there are more than one public payer for this intervention (eg, Government of Winnipeg, Government of Manitoba), we will conduct separate analyses for each relevant perspective to assess the 'value for money' specifically to each payer. We will characterise the uncertainty of our findings using a cost-effectiveness acceptability curve and 95\% CI. In addition, we will explore the possibility of building a decision analytic model that will examine the potential 
long-term economic impact of the intervention after the study period. ${ }^{72}$ This economic model will use various data sources to estimate costs including the study, ${ }^{73} 74$ published literature ${ }^{67-69}$ and expert opinion.

\section{DISCUSSION}

The proposed natural experimental study will fill a large gap in our understanding of the impacts of changes to the built environment on CVD-related outcomes. Specifically, we will provide critical experimental evidence for the impact of changes to urban spaces for recreational physical activity and active transportation on CVD-related morbidity and mortality. Additionally, to support evidence for future policies relating to urban activity corridors, we will also provide an estimate for the cost-effectiveness of large urban trail networks within cities.

\section{Author affiliations}

1Dalla Lana School of Public Health, University of Toronto, Toronto, Ontario, Canada

${ }^{2}$ Winnipeg Trails, Winnipeg, Manitoba, Canada

${ }^{3}$ Department of Endocrinology and Metabolism, University of Toronto, Toronto, Ontario, Canada

${ }^{4}$ Department of Pediatrics and Child Health, Faculty of Health Sciences, University of Manitoba, Winnipeg, Manitoba, Canada

${ }^{5}$ The Bartlett Centre for Advanced Spatial Analysis (CASA), University College London, London, UK

${ }^{6}$ Institute of Health Policy Management and Evaluation, University of Toronto, Toronto, Ontario, Canada

${ }^{7}$ Active Transportation, City of Winnipeg, Winnipeg, Manitoba, Canada

\section{Twitter Erin Hobin @erinhobinPhD and Laura C Rosella @LauraCRosella}

Contributors All authors contributed to the study and manuscript in alignment with current ICMJE guidelines. The study was conceived by JM, AS and EH. JM and $E H$ are the principal investigators on the original funded grant. EH, AS, GB, KR, LCR, BTS, WI and JM participated in designing the study and submitting the original grant. JM and SW were involved in recruiting the City of Winnipeg to share data. $\mathrm{EH}, \mathrm{AS}, \mathrm{KR}$ and JM designed the field data collection processes. EM is leading the geomapping of trail users and interpretation of gentrification data. WI is leading the cost-effectiveness analyses of the study, and SW is providing data for these analyses. AS, NB and JM are involved in data cleaning and verification. EH, NB and JM drafted the original manuscript. All authors contributed to critically revising the manuscript for important intellectual content, gave their final approval and agreed to be accountable for all aspects of the work and they will participate in future interpretation of the data and drafting of further manuscripts arising from this work.

Funding Funding for this project was provided by an operating grant from the Heart and Stroke Foundation of Canada (G-17-0018638) and the Canadian Institutes of Health Research (PJT-153449; CPP-137910).

Disclaimer Funding bodies were not involved in the study design, conduct, interpretation or manuscript preparation for this project.

Map disclaimer The depiction of boundaries on this map does not imply the expression of any opinion whatsoever on the part of BMJ (or any member of its group) concerning the legal status of any country, territory, jurisdiction or area or of its authorities. This map is provided without any warranty of any kind, either express or implied.

Competing interests None declared.

Patient consent for publication Not required.

Ethics approval This study was approved by the Biomedical Research Ethics Board at the University of Manitoba (H2017:232) and the Health Information Privacy Committee at the Winnipeg Regional Health Authority (HIPC - 2019/2020-05).

Provenance and peer review Not commissioned; peer reviewed for ethical and funding approval prior to submission.

Open access This is an open access article distributed in accordance with the Creative Commons Attribution Non Commercial (CC BY-NC 4.0) license, which permits others to distribute, remix, adapt, build upon this work non-commercially, and license their derivative works on different terms, provided the original work is properly cited, appropriate credit is given, any changes made indicated, and the use is non-commercial. See: http://creativecommons.org/licenses/by-nc/4.0/.

\section{ORCID iDs}

Laura C Rosella http://orcid.org/0000-0003-4867-869X

Jonathan McGavock http://orcid.org/0000-0002-3741-5248

\section{REFERENCES}

1 Sallis JF, Bull F, Guthold R, et al. Progress in physical activity over the Olympic quadrennium. Lancet 2016;388:1325-36.

2 Sallis JF, Floyd MF, Rodríguez DA, et al. Role of built environments in physical activity, obesity, and cardiovascular disease. Circulation 2012;125:729-37.

3 Meijer M, Röhl J, Bloomfield K, et al. Do neighborhoods affect individual mortality? A systematic review and meta-analysis of multilevel studies. Soc Sci Med 2012;74:1204-12.

4 Frank LD, Kavage S, Devlin A. Health and the built environment: a review, 2012.

5 McCormack GR, Shiell A. In search of causality: a systematic review of the relationship between the built environment and physical activity among adults. Int J Behav Nutr Phys Act 2011;8:125.

6 Ferdinand AO, Sen B, Rahurkar S, et al. The relationship between built environments and physical activity: a systematic review. Am J Public Health 2012;102:e7-13.

7 lo M. Bringing public health into urban Revitalization: workshop summary. bringing public health into urban Revitalization: workshop summary. Washington (DC), 2015.

8 Gascon M, Vrijheid M, Nieuwenhuijsen MJ. The built environment and child health: an overview of current evidence. Curr Environ Health Rep 2016;3:250-7.

9 Mayne SL, Auchincloss AH, Michael YL. Impact of policy and built environment changes on obesity-related outcomes: a systematic review of naturally occurring experiments. Obes Rev 2015;16:362-75.

10 Sallis JF, Cerin E, Conway TL, et al. Physical activity in relation to urban environments in 14 cities worldwide: a cross-sectional study. Lancet 2016;387:2207-17

11 Floyd MF, Spengler JO, Maddock JE, et al. Park-based physical activity in diverse communities of two U.S. cities. An observational study. Am J Prev Med 2008;34:299-305.

12 Li F, Harmer P, Cardinal BJ, et al. Obesity and the built environment: does the density of neighborhood fast-food outlets matter? Am J Health Promot 2009;23:203-9.

13 Polsky JY, Moineddin R, Glazier RH, et al. Relative and absolute availability of fast-food restaurants in relation to the development of diabetes: a population-based cohort study. Can J Public Health 2016;107:eS27-33.

14 Beale SJ, Bending MW, Trueman P, et al. Should we invest in environmental interventions to encourage physical activity in England? an economic appraisal. Eur J Public Health 2012;22:869-73.

15 Guo JY, Gandavarapu S. An economic evaluation of healthpromotive built environment changes. Prev Med 2010;50:S44-9.

16 Ding D, Gebel K. Built environment, physical activity, and obesity: what have we learned from reviewing the literature? Health Place 2012;18:100-5.

17 Abadie A, Diamond A, Hainmueller J. Synthetic Control Methods for Comparative Case Studies: Estimating the Effect of California's Tobacco Control Program. J Am Stat Assoc 2010;105:493-505.

18 Amatya A, Bhaumik D, Gibbons RD. Sample size determination for clustered count data. Stat Med 2013;32:4162-79.

19 Bancroft C, Joshi S, Rundle A, et al. Association of proximity and density of parks and objectively measured physical activity in the United States: a systematic review. Soc Sci Med 2015;138:22-30.

20 Clark S, Bungum T, Shan G, et al. The effect of a TRAIL use intervention on urban TRAIL use in southern Nevada. Prev Med 2014;67:S17-20.

21 Creatore MI, Glazier RH, Moineddin R, et al. Association of neighborhood Walkability with change in overweight, obesity, and diabetes. JAMA 2016;315:2211-20.

22 Finlayson GS, Lix LM, Roos LL. The whole is greater than the sum of the parts: using data linkage and cohort designs to create data synergy at MCHP. Health Policy 2011;6:83-5.

23 Fransoo RR, Martens PJ, Prior HJ, et al. The rising prevalence of asthma: true increase, diagnostic exchange or diagnostic accuracy? Healthc Policy 2013;8:27-34. 
24 Martens PJ. Straw into gold: lessons learned (and still being learned) at the Manitoba centre for health policy. Healthc Policy 2011;6:44-54.

25 Nickel NC, Chateau DG, Martens PJ, et al. Data resource profile: pathways to health and social equity for children (paths equity for children). Int J Epidemiol 2014;43:1438-49.

26 Roos LL, Soodeen R-A, Bond R, et al. Working more productively: tools for administrative data. Health Serv Res 2003;38:1339-58.

27 Smith M, Roos LL, Burchill C. Expanding the data Repository: new technology and resources for the 21 st century. Healthc Policy 2011;6:104-6.

28 Kontopantelis E, Doran T, Springate DA, et al. Regression based quasi-experimental approach when randomisation is not an option: interrupted time series analysis. BMJ 2015;350:h2750.

29 Sims M, Maxwell R, Bauld L, et al. Short term impact of smoke-free legislation in England: retrospective analysis of hospital admissions for myocardial infarction. BMJ 2010;340:c2161.

30 Jandoc R, Burden AM, Mamdani M, et al. Interrupted time series analysis in drug utilization research is increasing: systematic review and recommendations. J Clin Epidemiol 2015;68:950-6.

31 Clancy L, Goodman P, Sinclair H, et al. Effect of air-pollution control on death rates in Dublin, ireland: an intervention study. Lancet 2002;360:1210-4.

32 Law MR, Soumerai SB, Adams AS, et al. Costs and consequences of direct-to-consumer advertising for clopidogrel in Medicaid. Arch Intern Med 2009;169:1969-74.

33 McAlister FA, Bakal JA, Majumdar SR, et al. Safely and effectively reducing inpatient length of stay: a controlled study of the general internal medicine care transformation initiative. BMJ Qual Saf 2014;23:446-56.

34 Serumaga B, Ross-Degnan D, Avery AJ, et al. Effect of pay for performance on the management and outcomes of hypertension in the United Kingdom: interrupted time series study. BMJ 2011;342:d108.

35 EPOC-Cochrane. EPOC- criteria for including interrupted time series analyses, 1998. Available: http://epoccochraneorg/sites/ epoccochraneorg/files/uploads/inttimepdf

36 Ramsay CR, Matowe L, Grilli R, et al. Interrupted time series designs in health technology assessment: lessons from two systematic reviews of behavior change strategies. Int J Technol Assess Health Care 2003;19:613-23.

37 Wagner AK, Soumerai SB, Zhang F, et al. Segmented regression analysis of interrupted time series studies in medication use research. J Clin Pharm Ther 2002;27:299-309.

38 von Hippel P, Benson R. Obesity and the natural environment across us counties. Am J Public Health 2014;104:1287-93.

39 EPOC. EPaOoC. interrupted time series (its) analyses. Oslo: Norwegian Knowledge Centre for the Health Services, 2013.

40 Linden A, Adams JL. Applying a propensity score-based weighting model to interrupted time series data: improving causal inference in programme evaluation. J Eval Clin Pract 2011;17:1231-8.

41 Distasio J, Kaufman A. The divided Priarie City: income inequality among Winnipeg's neighbourhoods. Institute for Urban Studies, University of Winnipeg, 2015

42 Fransoo R, Martens P, Prior H, et al. The $2013 \mathrm{RHA}$ health indicators atlas, University of Manitoba, 2013. Available: http://mchp-appserv. cpe.umanitoba.ca/reference/RHA_2013_web_version.pdf

43 Lix LM, Yogendran MS, Shaw SY, et al. Population-Based data sources for chronic disease surveillance. Chronic Dis Can 2008;29:31-8.

44 Marrie RA, Fisk J, Tremlett $\mathrm{H}$, et al. Differing trends in the incidence of vascular comorbidity in MS and the general population. Neurol Clin Pract 2016;6:120-8.

45 Ermagun A, Lindsey G, Loh TH. Urban trails and demand response to weather variations. Transp Res D Transp Environ 2018;63:404-20.

46 Lix LM, Kuwornu JP, Kroeker K, et al. Estimating the completeness of physician billing claims for diabetes case ascertainment using population-based prescription drug data. Health Promot Chronic Dis Prev Can 2016;36:55-60.

47 Lindsey GPM, Hankey S. The Minnesota Bicycle and Pedestrian Counting Initiative: Implementation Study. In: Management MDoTOoTS. St. Paul, Minnesota: University of Minnesota, 2015.

48 Budowski A. Developing expansion factors to estimate cyclist seasonal average daily traffic in Winnipeg, MB. Winnipeg, Manitoba: University of Manitoba, 2015.

49 Chateau D, Metge $\mathrm{C}$, Prior $\mathrm{H}$, et al. Learning from the census: the socio-economic factor index (SEFI) and health outcomes in Manitoba. Can J Public Health 2012;103:S23-7.

50 Lees L, Slater T, Wyly E. The birth of Gentrification. In: Gentrification. New York, NY: Taylor and Francis Group, LLC, 2013: 3-38.
51 Kavanagh A, Bentley RJ, Turrell G, et al. Socioeconomic position, gender, health behaviours and biomarkers of cardiovascular disease and diabetes. Soc Sci Med 2010;71:1150-60.

52 Mackenbach JP, Cavelaars AE, Kunst AE, et al. Socioeconomic inequalities in cardiovascular disease mortality; an international study. Eur Heart J 2000;21:1141-51.

53 Winkleby MA, Jatulis DE, Frank E, et al. Socioeconomic status and health: how education, income, and occupation contribute to risk factors for cardiovascular disease. Am J Public Health 1992;82:816-20.

54 Lysy Z, Booth GL, Shah BR, et al. The impact of income on the incidence of diabetes: a population-based study. Diabetes Res Clin Pract 2013;99:372-9.

55 Lipscombe LL, Austin PC, Manuel DG, et al. Income-Related differences in mortality among people with diabetes mellitus. CMAJ 2010;182:E1-17.

56 Brook JR, Setton EM, Seed E, et al. The Canadian Urban Environmental Health Research Consortium - a protocol for building a national environmental exposure data platform for integrated analyses of urban form and health. BMC Public Health 2018;18:114.

57 Pattanayak CW, Rubin DB, Zell ER. [Propensity score methods for creating covariate balance in observational studies]. Rev Esp Cardiol 2011;64:897-903.

58 Donner A, Klar N. Design and analysis of cluster randomization trials in health research. London, ON: Arnold, 2000.

59 Fransoo R, Martens P, Burland E. The need to know team. Manitoba RHA indicators atlas 2009. Elnnipeg, MB: University of Manitoba, 2009.

60 Pell JP, Haw S, Cobbe S, et al. Smoke-Free legislation and hospitalizations for acute coronary syndrome. $N$ Engl J Med 2008;359:482-91.

61 MacDonald JM, Stokes RJ, Cohen DA, et al. The effect of light rail transit on body mass index and physical activity. Am J Prev Med 2010;39:105-12.

62 Schaefer L, Plotnikoff RC, Majumdar SR, et al. Outdoor time is associated with physical activity, sedentary time, and cardiorespiratory fitness in youth. J Pediatr 2014;165:516-21.

63 Shephard RJ, Aoyagi Y. Seasonal variations in physical activity and implications for human health. Eur J Appl Physiol 2009;107:251-71.

64 Danet S, Richard F, Montaye M, et al. Unhealthy effects of atmospheric temperature and pressure on the occurrence of myocardial infarction and coronary deaths. A 10-year survey: the Lille-World health organization MONICA Project (monitoring trends and determinants in cardiovascular disease). Circulation 1999;100:E1-7

65 Group TE. Cold exposure and winter mortality from ischaemic heart disease, cerebrovascular disease, respiratory disease, and all causes in warm and cold regions of Europe. The Eurowinter group. Lancet 1997;349:1341-6.

66 DR BPJ. Introduction to time series and forecasting. 2nd ed. New York, NY: Springer, 2001

67 Tricco AC, Cogo E, Isaranuwatchai W, et al. A systematic review of cost-effectiveness analyses of complex wound interventions reveals optimal treatments for specific wound types. BMC Med 2015;13:90.

68 Marsh JD, Birmingham TB, Giffin JR, et al. Cost-Effectiveness analysis of arthroscopic surgery compared with non-operative management for osteoarthritis of the knee. BMJ Open 2016;6:e009949.

69 Tricco AC, Ashoor HM, Antony J, et al. Safety, effectiveness, and cost effectiveness of long acting versus intermediate acting insulin for patients with type 1 diabetes: systematic review and network meta-analysis. BMJ 2014;349:g5459.

70 Hoch JS, Briggs AH, Willan AR. Something old, something new, something borrowed, something blue: a framework for the marriage of health econometrics and cost-effectiveness analysis. Health Econ 2002;11:415-30.

71 Willan AR, Briggs $\mathrm{AH}$, Hoch JS. Regression methods for covariate adjustment and subgroup analysis for non-censored costeffectiveness data. Health Econ 2004;13:461-75.

72 Drummond MF, Sculpher MJ, Claxton K, et al. Methods for the economic evaluation of health care programmes. Oxford, UK: Oxford university press, 2015.

73 Cui Y, Doupe M, Katz A, et al. Economic evaluation of Manitoba health lines in the management of congestive heart failure. Healthc Policy 2013;9:36-50.

74 Forget EL. New questions, new data, old interventions: the health effects of a guaranteed annual income. Prev Med 2013;57:925-8. 